\title{
O PROFISSIONAL DE ENFERMAGEM E A QUALIDADE DE VIDA: UMA ABORDAGEM FUNDAMENTADA NAS DIMENSÕES PROPOSTAS POR FLANAGAN
}

Rosemery Andrade Lentz* Regina G. Santini Costenaro**

Lúcia H.T. Gonçalves***

Sílvia M. Nassar****

LENTZ, R.A; COSTENARO, R.G.S.; GONÇALVES, L.H.T.; NASSAR, S.N. O profissional de enfermagem e a qualidade de vida: uma abordagem fundamentada nas dimensões propostas por Flanagan. Rev.latinoam.enfermagem, Ribeirão Preto, v. 8, n. 4, p. 7-14, agosto 2000.

Estudo quali-quantitativo que utilizando a Escala de Qualidade de Vida de Flanagan objetivou verificar significado(s) de qualidade de vida para os profissionais de enfermagem; identificar as dimensões da qualidade de vida mais valorizadas pelos mesmos e explorar relação entre escores de qualidade de vida obtidos pelos profissionais de Unidade DST/AIDS e UTI neonatal. Os dois grupos mostraram resultados similares quanto aos significados e dimensão de qualidade de vida mais valorizada. Os escores totais apresentaram uma avaliação positiva da qualidade de vida dos dois grupos, entretanto demostraram diferença no nivel de qualidade de vida entre as duas equipes de enfermagem.

UNITERMOS: qualidade de vida, equipe de enfermagem

\section{INTRODUÇÃO}

À véspera do século XXI e do terceiro milênio, vivemos um momento de efervescência tecnológica e da constatação de que a tecnologia não dá conta das necessidades do ser humano. Pesquisas e estudos sobre qualidade de vida (QV) tem crescido significativamente nos últimos anos, mostrando a preocupação dos pesquisadores em suprir o que o avanço tecnológico não foi capaz de fazer.

Muitos termos são usados na literatura como sinônimos de qualidade de vida, tais como bem-estar, felicidade, boas condições de vida e satisfação na vida. MEEBERG (1993), apresenta o estudo realizada por Walcker \& Avant onde estes relacionam os atributos que se incluem quando o conceito de qualidade de vida é descrito ou definido, quais sejam: a) um sentimento de satisfação com a própria vida em geral; b) capacidade mental de avaliar sua própria vida como satisfatória; c) um estado aceitável de saúde física, emocional, mental e social; d) uma avaliação objetiva feita por outro que as condições de vida da pessoa são adequadas e não ameaçadoras à vida.

A qualidade de vida é uma dimensão complexa para ser definida e sua conceituação, ponderação e valorização vêm sofrendo uma evolução, que por certo acompanha a dinâmica da humanidade, suas diferentes culturas, suas prioridades e crenças. Para ROMANO (1993), qualidade de vida é mais que simplesmente a ausência ou presença de saúde, abrangendo também educação, saneamento básico, acesso a serviços de saúde, satisfação e condições de trabalho, além de outros aspectos.

Considerando a escassez em nosso meio de pesquisas que abordam a qualidade de vida dos profissionais de enfermagem que prestam assistência

\footnotetext{
* Enfermeira do Hospital Regional de São José Dr. Homero de Miranda Gomes, docente da UNISUL, mestre em enfermagem/ UFSC, doutoranda em enfermagem/UFSC

** Enfermeira do Hospital Universitário de Santa Maria, docente das Faculdades Franciscanas/SM, mestre em educação, doutoranda em enfermagem/UFSC

*** Docente do Departamento de Enfermagem/UFSC, coordenadora do GESPI/PEN - Grupo de estudos de cuidados de saúde de pessoas idosas

**** Docente do Departamento de Ciências Estatísticas e da Computação/UFSC, assessora estatística do GESPI/PEN
} 
especializada, e o desconhecimento do seu nível de satisfação buscamos neste estudo explorar esta lacuna existente. Assim, pontuamos os seguintes objetivos: a) verificar o(s) significado(s) de qualidade de vida para os profissionais de enfermagem; b) identificar as dimensões da qualidade de vida, segundo a Escala de Qualidade de Vida de Flanagan (EQV), mais valorizadas pelos profissionais de enfermagem; c)explorar a relação existente entre os escores de qualidade de vida obtidos pelos profissionais da enfermagem atuantes em uma unidade de terapia neonatal (UTI neonatal) e em uma unidade de doenças sexualmente transmissíveis e AIDS (DST/AIDS), segundo a Escala de Qualidade de Vida de Flanagan(EQV).

\section{METODOLOGIA}

O estudo de natureza quali-quantitativo, foi realizado junto à equipe de enfermagem de duas unidades de internação, em dois hospitais públicos, gerais e de grande porte, um na cidade de Florianópolis-SC e o outro na cidade de Santa Maria-RS abrangendo respectivamente a unidade de internação DST/AIDS e UTI neonatal.

Fizeram parte da amostra trinta e sete (37) profissionais de enfermagem de UTI neonatal, amostra que denominamos aqui Grupo T e vinte e dois (22) da unidade DST/AIDS, denominada Grupo A e, cujos participantes selecionados aceitaram espontaneamente colaborar com a pesquisa; após informados de todos os cuidados éticos. O procedimento para coleta de dados, constou da distribuição da EQV de Flanagan (Anexo 1), aos participantes para auto-aplicação e recolhimento posterior. Solicitou-se também preencher alguns dados relativos à identificação como: idade, sexo, categoria profissional, estado civil; assim como responder a uma questão aberta sobre o significado dado à qualidade de vida.

A escala de qualidade de vida utilizada foi a proposta por FLANAGAN (1982) trata-se de uma escala psicométrica de natureza ordinal em cuja aplicação obtém-se respostas em quinze itens agrupados em cinco dimensões: bem estar físico e material; relações com outras pessoas; atividades sociais, comunitárias e cívicas; desenvolvimento pessoal e realização; e, recreação. FLANAGAN (1982) desenvolveu esta escala com os dados colhidos de uma amostra de três mil homens e mulheres norte-americanos, com idades de 30, 50 e 70 anos, utilizando o método de incidente crítico. Foi considerado um instrumento multidimensional e com evidência de validade de construto em função dos resultados da análise fatorial feita. BURCKHARDT et al. (1989) utilizou-a modificando a original graduação de respostas de cinco pontuações para sete pontuações por permitir um espectro maior de respostas.

Para o presente estudo foi utilizado EQV de Flanagan com pontuação adotada por BURCKHARDT et al. (1989) cujas respostas vão de muitíssimo satisfeito com valor sete até muitíssimo insatisfeito com valor um. Assim, a pontuação máxima que pode ser obtida na EQV é de cento e cinco, representando escore total de mais alta qualidade de vida, e a pontuação mínima de quinze, representando escore total de mais baixa qualidade de vida.

Esta escala foi traduzida para o português e usada por HASHIMOTO et al. (1996), os quais porém, não fazem referência quanto a sua fidedignidade. Por isso, GONÇALVES \& NASSAR (1998)***** calcularam o coeficiente de fidedignidade reunindo os dados de aplicação da EQV de Flanagan de três amostras diferentes: uma de 72 pacientes idosos em tratamento ambulatorial, uma de 52 pessoas freqüentadoras de uma universidade aberta para a terceira idade e uma de 59 profissionais da enfermagem, trabalhadores de uma UTI neonatal e unidade de pacientes com DST/AIDS, num total de 183 sujeitos. Dos dados desta amostra relativamente heterogênea demonstraram através de testes estatísticos que a EQV de Flanagan traduzida em português e aplicada em nosso meio apresenta alta fidedignidade.

\section{ANÁLISE E DISCUSSÃO DOS RESULTADOS}

Foram entrevistados, cinqüenta e nove (59) profissionais de enfermagem, sendo vinte e dois (22) da unidade de DST/AIDS e trinta e sete (37) da UTI neonatal. As características dos entrevistados são apresentadas na Tabela 1.

***** GONÇALVES, L.H.T.; NASSAR, S.M. - Teste de confiabilidade de EQV de Flanagan. Florianópolis, UFSC, 1998 ( não publicado). Maiores informações: email pen@repensul.ufsc.br 
Tabela 1 - Distribuição das características dos profissionais de enfermagem segundo os Grupos A (atuantes em unidade DST/AIDS) e T(em unidade UTI neonatal)

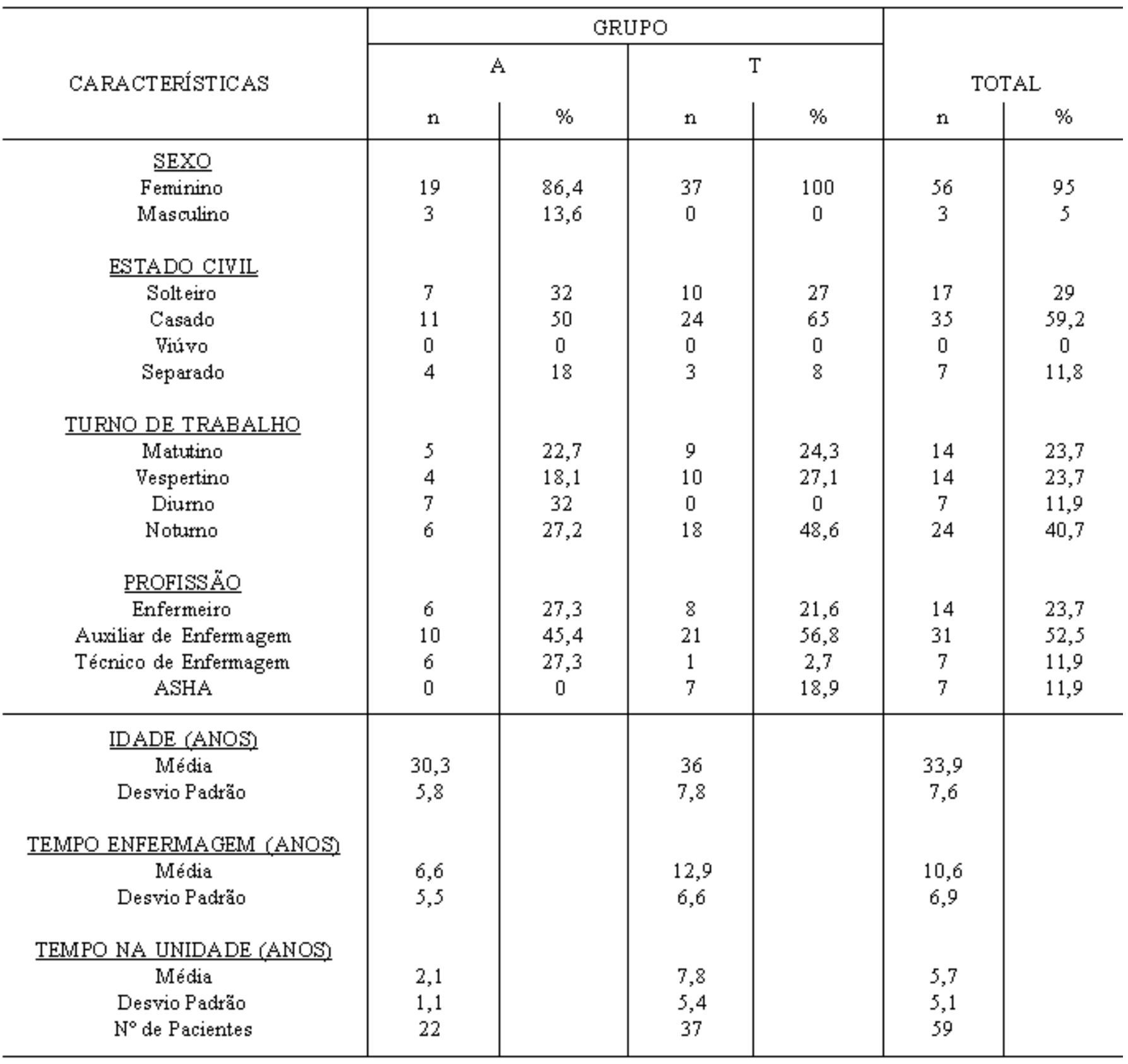

O total da população estudada apresentou um escore médio de qualidade de vida de 80,47 e um desvio padrão de 8,87 . Dos vinte e dois (22) profissionais entrevistados, pertencentes ao Grupo A, o que apresentou maior pontuação na Escala de QV de Flanagan atingiu escore total de 193 e o que apresentou mais baixa QV atingiu 62. O escore moda de QV encontrado foi de 72, apresentado por 3 entrevistados, o escore médio foi de 76 e o desvio padrão de 7,10 . Já no grupo dos trinta e sete (37) trabalhadores da UTI neonatal, o que apresentou maior escore na EQV de Flanagan, atingiu 96 pontos e o que apresentou menor índice de QV alcançou escore igual a 57. O escore moda de QV apresentado foi de 87, que representou a somatória de quatro (4) indivíduos e o escore médio encontrado foi de 83,14 , apresentando um desvio padrão de 8,82 .

Os dados apresentados mostram uma maior conformidade do nível de qualidade de vida do grupo A e uma maior variação do grupo T. Aplicando-se o teste não-paramétrico de Kolmogorov-Sminorv, obteve-se uma diferença máxima negativa de $-0,47$, e diferença máxima positiva de 0,03 resultando um valor de $\mathrm{p}<0,005$. Este nível de significância $\mathrm{p}<0,005$, nos leva a concluir que há diferença no nível de qualidade de vida dos dois grupos. 


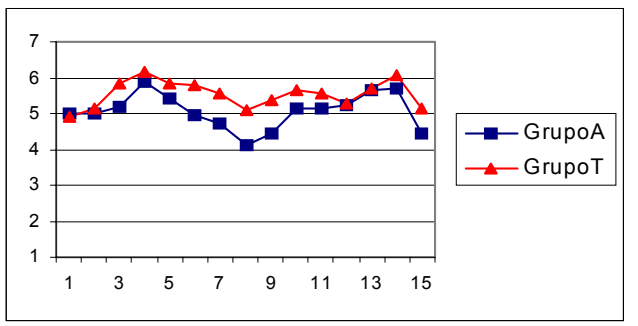

Figura 1 - Escores médios desvios-padrão em cada um dos itens da EQV de Flanagan obtidos nos Grupos A (atuantes em unidade DST/AIDS) e T (em unidade de UTI neonatal)

A Figura 1, permite-nos uma visualização do desempenho dos dois grupos, onde o grupo T apresenta escores mais altos de qualidade de vida do que o Grupo A, ou seja, entre os profissionais da UTI neonatal. Tal resultado leva-nos a conjecturar algumas possibilidades: O tipo de trabalho onde há expectativa de vida de crianças influencia na melhor qualidade de vida dos profissionais de enfermagem? Os escores de qualidade de vida mais altos, apresentados pelos profissionais de enfermagem da UTI neonatal, teriam relação com a satisfação pelo setor, quando examinado o tempo de atuação do mesmo? Já os escores de mais baixa qualidade de vida da equipe de enfermagem da unidade DST/AIDS relativos as dimensões Atividades sociais, comunitárias e cívicas e Recreação, poderiam ser explicados pela característica de idade mais jovem, época em que a luta pela sobrevivência pessoal e profissional prevalece?
A Tabela 2 mostra-nos que os dois grupos A e T, apresentaram escores de mais qualidade de vida na dimensão Relações com Outras Pessoas, mais especificamente no item Ter e criar filhos, no qual o escore médio encontrado foi de 5,86 e 6,16 respectivamente. O fato de possuir família e ocupar-se de sua prole, parece-nos ser importante para dotar o profissional de enfermagem de condições de maiores possibilidades de satisfação em sua vida pessoal/ profissional.

O escore mais baixo, apareceu nos dois grupos, na dimensão Atividades Sociais, Comunitárias e Cívicas, no item Participar em Associações e Atividades de Interesse Público, com o escore de 4,14 para o Grupo A e 5,08 para o T. A participação é o "caminho natural para o homem exprimir sua tendência inata de realizar, fazer coisas, afirmar-se a si mesmo..."(BORDENAVE, 1981, p.16), portanto é atributo fundamental da qualidade de vida.

Em relação ao significado de qualidade de vida, foi expressado pelos funcionários da equipe de enfermagem entre eles quatorze (14) enfermeiros, sete (07) técnicos de enfermagem, trinta e um (31) auxiliares de enfermagem e sete (07) atendentes de enfermagem, de ambos os grupos caracterizaram-na como de bem estar físico e material enfatizando os seguintes aspectos: ter bom emprego incluindo salário satisfatório; de ter lazer; de ter saúde; de ter alimentação/ moradia/ vestuário/ transporte; de ter educação e segurança. Não mencionaram atributos diferentes entre os dois grupos.

Tabela 2 - Escores médios desvios-padrão em cada um dos quinze ítens de EQV de Flanagan obtidos no grupo A e grupo $T$

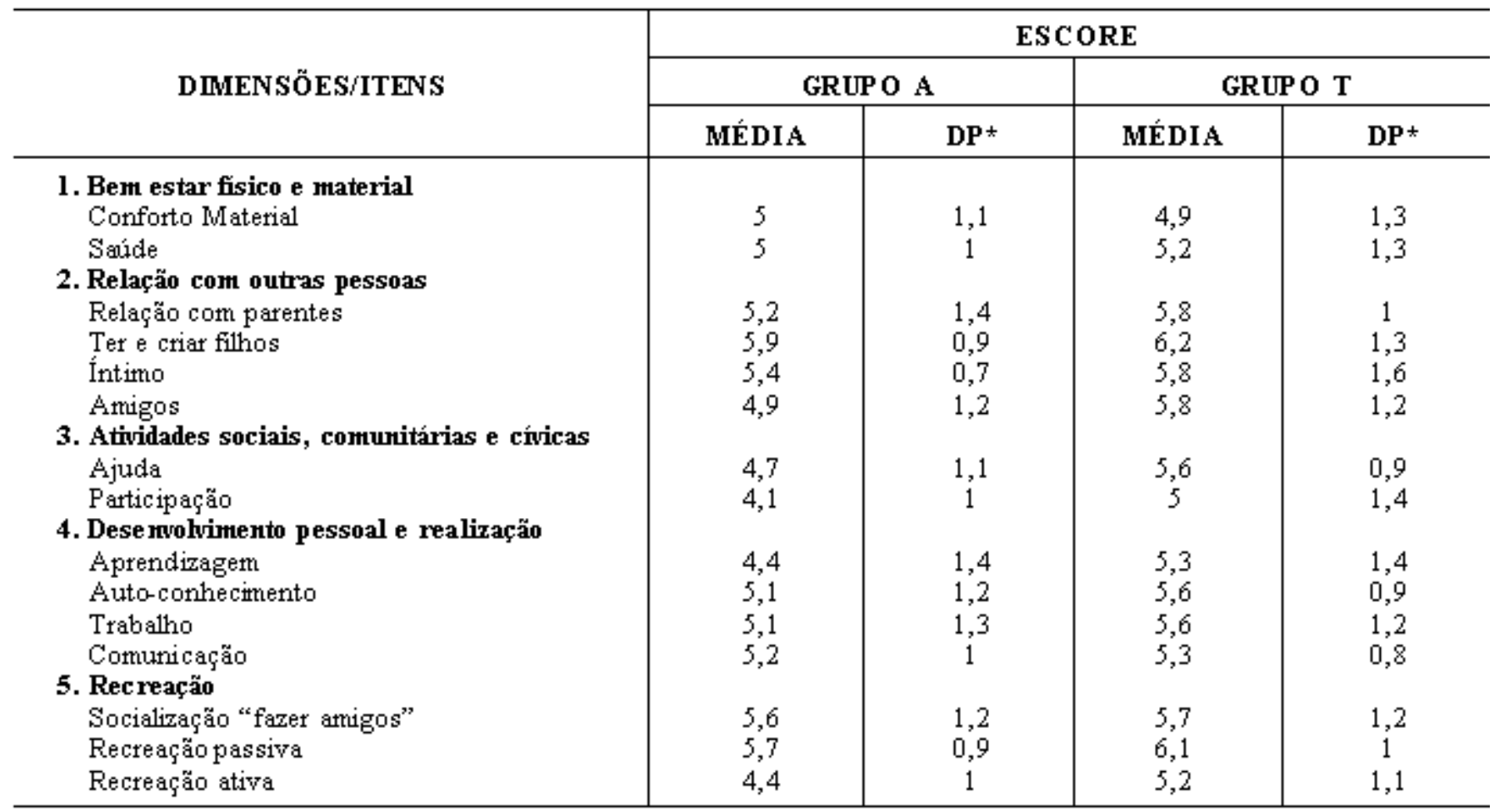


De acordo com a Constituição Brasileira (BRASIL. Constituição, 1988, p.20), que prescreve no capítulo II, referindo-se aos direitos sociais em seu Art. $6^{\circ}$ "São direitos sociais a educação, a saúde, o trabalho, o lazer, a segurança, a previdência social, a proteção a maternidade e a infância, a assistência aos desamparados, na forma desta Constituição". São considerados princípios inquestionáveis, ou seja, são necessidades vitais do ser humano a educação, a saúde, o transporte, o lazer e a seguridade social. Estes fatores parecem ser o mínimo necessário para que o ser humano leve uma vida digna. Na verdade a Constituição Brasileira reconhece o direito as necessidades e a igualdade para os seres humanos porém não constitui seu papel garantir a satisfação destas necessidades para todos.

Conviver com a família, ter amigos e manter um bom relacionamento social com as outras pessoas também são situações, expressadas pelos entrevistados, que interferem na Qualidade de Vida. DALY et al. (1996) escreve sobre a importância da família e dos amigos como também do sentimento que envolve este tipo de relacionamento. A equipe de enfermagem deve ser estimulada a manter uma relação de interação com os demais membros uma vez que a convivência social contribui para o desenvolvimento interior do ser humano, pois não vivemos totalmente independente, e por esta razão se torna necessário e relevante uma participação social.

PARSE (1994) escreve que alguns escritores se referem a qualidade de vida como sendo uma situação de status funcional e desempenho de funções; outros escritores incluem aspectos como enfrentamento, habilidade, estilo de vida e auto-estima; e um terceiro grupo indica que a qualidade de vida está relacionada ao nível de satisfação de necessidades.

A participação dos profissionais de enfermagem em associações, contribui para o processo de transformação, sendo através das atitudes ou mostrando por meio de suas crenças que é necessário mudar a coparticipação do profissional, do cliente e da instituição. Esta expansão da compreensão do fazer ilumina aspectos da qualidade de vida e da qualidade de saúde.
Outro ponto importante que surgiu fazendo parte da qualidade de vida da clientela em estudo, foi relacionado a valorização, reconhecimento, desenvolvimento e realização pessoal e profissional. Segundo DALY et al. (1996), muitos pesquisadores tem conceituado qualidade de vida como um indicador global de bem estar e satisfação de vida. Por isso podemos dizer que a qualidade de vida é impar, ou seja, tem múltiplas facetas e se apresenta de diferentes maneiras dependendo de como cada ser humano percebe ou vivência o seu cotidiano. A valorização, o desenvolvimento e o reconhecimento são fundamentais para a realização pessoal e vice-versa. Estes complementam o ser e o fazer na enfermagem, portanto deve ser preocupação das gerências de enfermagem enfatizar o profissional e pessoal de cada trabalhador desta equipe. A valorização e o reconhecimento dependem das outras pessoas ou seja, das que possuem cargos mais elevados e também dos que usufruem do fazer profissional. A realização depende do auto-conhecimento, do sentir profissional, da satisfação sentida em desenvolver seu trabalho, pois o profissional apresenta além do fazer, o ser que existe nele mesmo, não existe receita para isto, existe sim, um desprender-se de valores pré determinados. Assim, a realização depende também da valorização, do desenvolvimento e do reconhecimento que é oferecido através de uma relação humanística influenciando na qualidade de vida dos profissionais da enfermagem. As atividades devem ser desenvolvidas com prazer e não apenas por obrigação, e para melhor visualização desenhamos uma representação de tal idéia na Figura 2.

FERRANS (1990) expressa cinco categorias que podem iluminar a qualidade de vida que são: levar uma vida normal; ter felicidade e satisfação; alcançar os objetivos pessoais; manter a capacidade física e mental de maneira natural, e preservar a habilidade social. Não existe parâmetros que predominam para formação de normas de aferição da qualidade de vida, uma vez, que esta pode se apresentar diferente para cada ser humano, já que este ser é singular e a felicidade para um pode não ser para o outro e assim por diante.

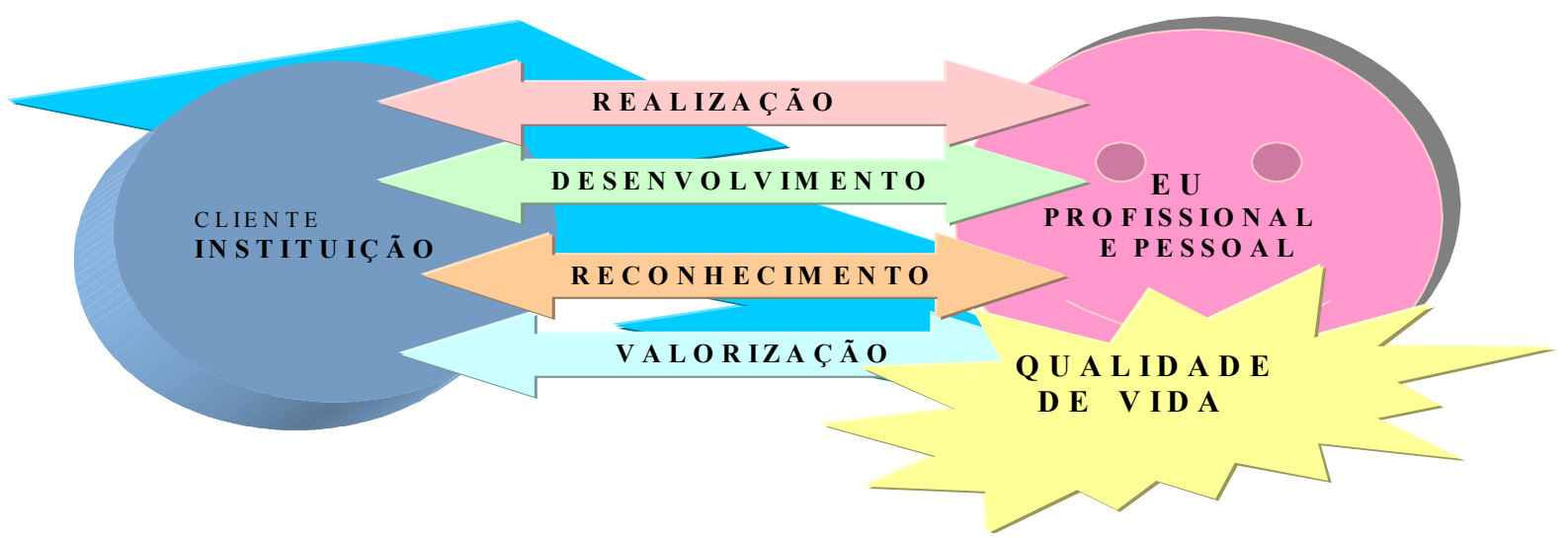

Figura 2 - Representação dos fatores que influenciam na qualidade de vida dos profissionais de enfermagem (Lentz, Costenaro - 1998) 
A enfermagem, se acreditar na teoria da transformação humana e a colocar em prática, passará a considerar a qualidade de vida como principal foco. A qualidade de vida além de ser ímpar, também é temporal pois a vivência de uma determinada situação pode ou não proporcionar uma qualidade de vida momentânea ou por um período mais longo. Portanto, quem pode definir a qualidade de vida de uma pessoa é ela própria, vivendo e interconectando o interior com o exterior. A interconexão com o ambiente, família, sociedade pode causar mudanças relevantes na vida do profissional da equipe de enfermagem.

A clientela em estudo caracteriza também como atributos de qualidade de vida a vivência divertida tendo um bem estar físico, mental e social; uma boa vida espiritual; ter paz, ter amigos e ser feliz. A saúde física, satisfação, a recreação e a felicidade são indicadores de bem estar, acoplam-se a outros fatores como ter paz e um bom relacionamento espiritual. A vivência divertida pode ocorrer de acordo com os respondentes, através de leituras, esportes, música, diálogo, etc. Todos permeiam uma relação em que o ser humano sadio ou doente deseja vivenciar, podendo este desejo ser caracterizado como biológico e inerente ao ser humano.

\section{CONSIDERAÇÕES GERAIS}

Os resultados deste estudo, sem ter a pretensão de estabelecer conclusão definitiva, pretendem colaborar para aumentar a compreensão de ser o conceito de qualidade de vida bastante complexo e que, nenhum modelo, conceito ou grau pode ser aplicado a todas as situações, a qualquer tempo, ou sempre.

Ao analisarmos os conceitos de qualidade de vida emitidos pelos dois grupos verificamos similaridades entre os atributos conceituados pelos mesmos, o que pode significar que independentemente do tipo de cliente que os profissionais de enfermagem cuidam, há concordância sobre o que consideram fundamentais para a seu bem viver.
A dimensão da qualidade de vida, segundo a Escala de Flanagan, mais valorizada pelos dois grupos A e T, foi a dimensão Relações com Outras Pessoas, mais especificamente no item Ter e criar filhos, onde o escore médio encontrado foi de 5,86 e 6,16 respectivamente. $\mathrm{O}$ escore mais baixo, apareceu nos dois grupos, na dimensão Atividades Sociais, Comunitárias e Cívicas, no item Participar em Associações e Atividades de Interesse Público, com o escore de 4,14 para o Grupo A e 5,08 para o $\mathrm{T}$.

Os escores totais de qualidade de vida obtidos pelos profissionais da enfermagem atuantes em uma UTI neonatal e em uma unidade de DST/AIDS, segundo a Escala de Qualidade de Vida de Flanagan (EQV), estão em sua grande maioria (58 dos 59 profissionais) acima de 60 , ou seja, acima da faixa de neutralidade ou indiferença, com tendências à valoração positiva. Contudo o resultado do teste não-paramétrico de Kolmogorov-Sminorv, aplicado nos escores de qualidade de vida de ambos os grupos apresentou nível de significância $\mathrm{p}<0,005$, o que nos leva a concluir que há diferença no nível de qualidade de vida dos dois grupos. Estes escores demonstram a diferença entre a equipe de enfermagem que trabalha na UTI neonatal e a que atua em unidade DST/AIDS, sugerindo a necessidade de estudos aprofundados de qualidade de vida dos profissionais de enfermagem segundo as áreas de atuação.

Portanto, enquanto profissionais, que somos temos que nos preocupar em valorizar o ser e o fazer da enfermagem proporcionando uma qualidade de vida satisfatória a estes trabalhadores. Referimos isto tanto a enfermagem assistencial que abrange a promoção e a recuperação da saúde como também a academia a qual possui participação relevante na formação de profissionais desta equipe.

AGRADECIMENTOS: Aos participantes da pesquisa, que gentilmente consentiram em colaborar com o presente estudo e as bolsistas de IC/CNPq Michele M.Dias e Tatiana G. de Liz pela organização da planilha dos dados.

\section{NURSING PROFESSIONALS AND QUALITY OF LIFE: AN APPROACH BASED ON THE DIMENSIONS PROPOSED BY FLANAGAN}

The present qualitative and quantitative study had the purposes to verify the meaning of quality of life to nursing professionals using Flanagan's Scale, to identify the quality of life dimensions that are most valued by those professionals and to explore the relations between the quality of life scores obtained by the professionals working at the STD/AIDS unit and at the neonatal ICU. The two groups showed similar results with respect to the meanings and the most valued dimension of quality of life. The total scores presented a positive evaluation of quality of life by the groups, although they showed a difference in the level of quality of life experienced by the two nursing teams. 


\section{LOS PROFESIONALES DE ENFERMERÍA Y LA CALIDAD DE VIDA: UN ABORDAJE BASADO EN LAS DIMENSIONES PROPUESTAS POR FLANAGAN}

Estúdio cuali-cuantitativo que usando la Escala de Calidad de Vida de Flanagan, pretende verificar significado(s) de calidad de vida para los profesionales de enfermería; identificar las dimensiones de la calidad de vida más valorizada por ellos mismos y explorar la relación entre los puntages de calidad de vida, obtenidos por los profesionales de la Unidad DST/SIDA y neonatal de CTI. Los dos grupos presentaron resultados similares con relación a los significados de calidad de vida y dimensión de calidad de vida más valorada. Los "escores" totales presentaron una evaluación positiva de calidad de vida de los dos grupos, sin embargo demostraron diferencia en el nivel de calidad de vida entre los dos equipos de enfermería.

TÉRMINOS CLAVES: calidad de vida, equipo de enfermería

\section{ANEXO 1}

\section{Escala de Qualidade de vida de Flanagan - EQV}

A escala EQVF busca avaliar a qualidade de vida utilizando as seguintes expressões lingüísticas:

9

$\odot$

\begin{tabular}{|c|c|c|c|c|c|c|}
\hline Muito Insatisfe ito & Insatisfe ito & Pouco Insatis feito & Indife rente & Pouco Satisfeito & Satis feito & Muito Satisfeito \\
\hline 1 & 2 & 3 & 4 & 5 & 6 & 7 \\
\hline
\end{tabular}

Às expressões lingüísticas são atribuídos escores numa faixa de 1 a 7 pontos, conforme indicado acima. Responda cada um dos itens abaixo assinalando o escore que indica seu grau de satisfação em relação aos seguintes aspectos de sua vida:

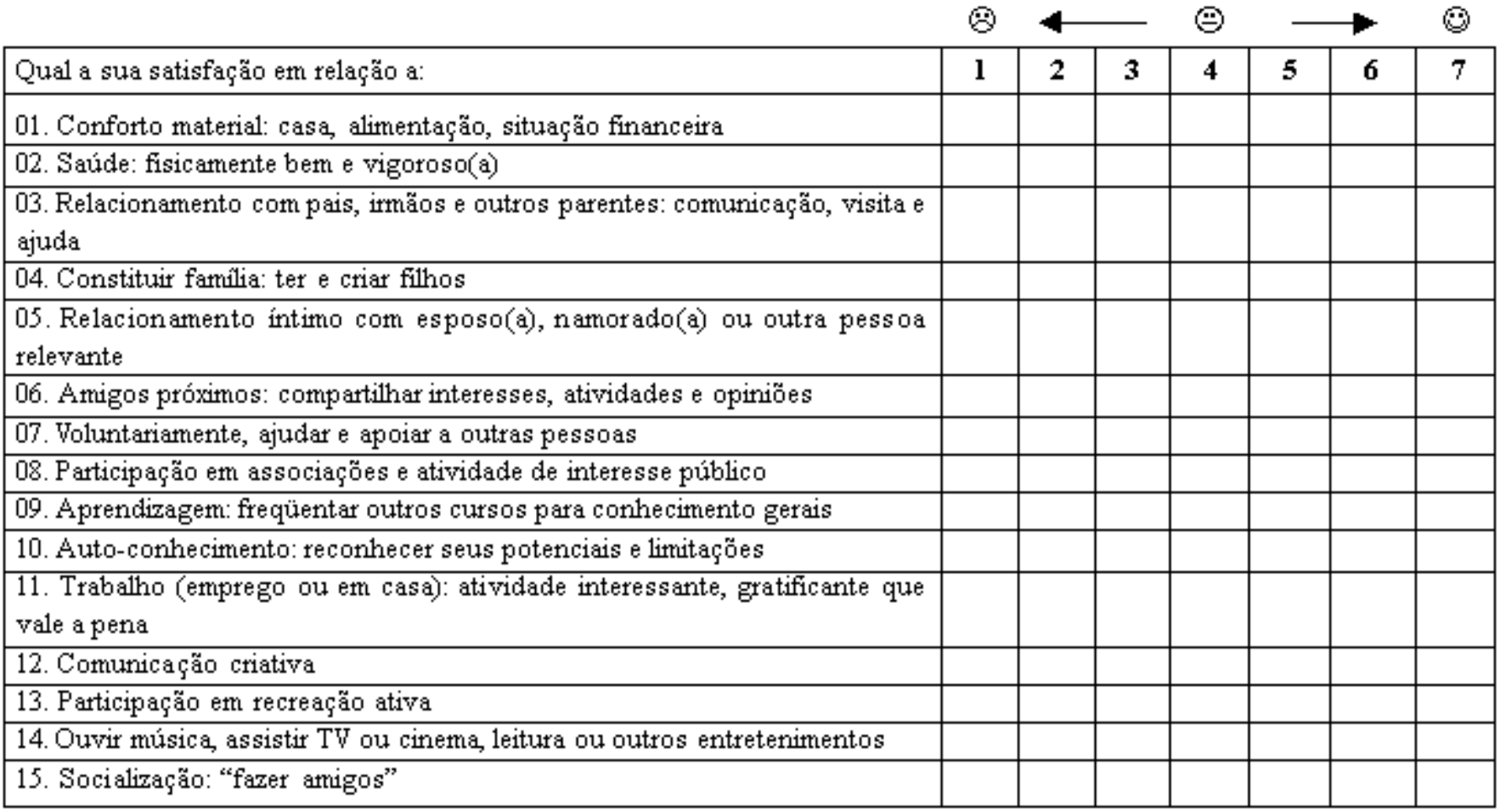

Utilize o verso desta folha, se desejar, para falar sobre QUALIDADE DE VIDA PARA VOCÊ:

Dimensões da escala de Flanagan

\begin{tabular}{|l|c|}
\hline \multicolumn{1}{|c|}{ Dimensões da EQVF } & ITENS \\
\hline 1. Bem estar fisico e material & 1 e 2 \\
\hline 2. Relações com outras pessoas & $3,4,5$ e 6 \\
\hline 3. Atividades sociais, comunitárias e cívicas & 7 e 8 \\
\hline 4. Desenvolvimento pessoal e realização & $9,10,11$ e 12 \\
\hline 5. Recreação & 13,14 e 15 \\
\hline
\end{tabular}




\section{REFERÊNCIAS BIBLIOGRÁFICAS}

01. BORDENAVE, J.E.D. O que é participação? São Paulo: Brasiliense, 1981.

02. BRASIL. Constituição da República Federativa do Brasil. Brasília: Centro Gráfico, 1988.

03. BURCKHARDT, C.S. et al. Quality of life of adults with chronic Illness: a psychometric study. Rev. Nurs. \& Health., v. 12, p. 347-354, 1989.

04. DALY, J. et al. Quality of life and the human becoming theory exploring disicpline-specific contributions. Nurs. Sci. Q., v. 9, n. 4, p. 70-74, 1996.

05. FERRANS, C.E. Quality of life: conceptual issues: seminars in oncology. Nursing, v. 6, p. 248-254, 1990.
06. FLANAGAN, J.C. Measurement of quality of life: current state of the art. Arch. Phys. Med. Rehabil., v. 63, p. 56-59, Feb.1982.

07. HASHIMOTO, H.; GUEDES, S.L.; PEREIRA, V.C. O ostomizado e a qualidade de vida: abordagem fundamentada nas dimensões da qualidade de vida proposta por Flanagan. São Paulo, 1996. (Monografia de conclusão do VII Curso de Especialização em Enfermagem em Estomatoterapia) - Escola de Enfermagem, Universidade de São Paulo.

08. MEEBERG, G.A. Quality of life: a concept analysis. J. Adv.Nurs., v. 18, p. 32-38,1993.

09. PARSE, R.R. Quality of life: sciencing and living the art of human becoming. Sci. Q., v. 7, n. 1, p. 1621, 1994.

10. ROMANO, B.W. Qualidade de vida: teoria e prática. Rev. Soc. Cardiol., São Paulo, v. 3,n. 6, p. 6-9, 1993. 